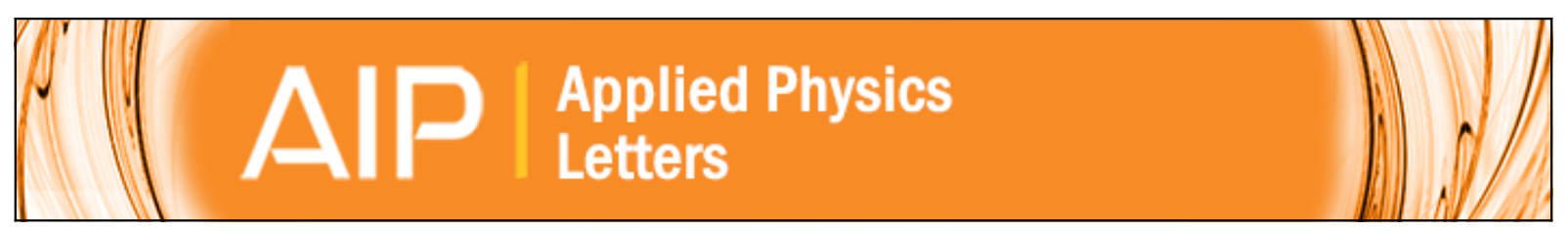

High mobility of the strongly confined hole gas in AgTaO3/SrTiO3

S. Nazir, M. Upadhyay Kahaly, and U. Schwingenschlögl

Citation: Applied Physics Letters 100, 201607 (2012); doi: 10.1063/1.4719106

View online: http://dx.doi.org/10.1063/1.4719106

View Table of Contents: http://scitation.aip.org/content/aip/journal/apl/100/20?ver=pdfcov

Published by the AIP Publishing 


\title{
High mobility of the strongly confined hole gas in $\mathrm{AgTaO}_{3} / \mathrm{SrTiO}_{3}$
}

\author{
S. Nazir, M. Upadhyay Kahaly, and U. Schwingenschlögla $\left.\right|^{\text {a) }}$ \\ KAUST, Physical Science and Engineering Division, Thuwal 23955-6900, Kingdom of Saudi Arabia
}

(Received 14 December 2011; accepted 3 May 2012; published online 18 May 2012)

\begin{abstract}
A theoretical study of the two-dimensional hole gas at the $(\mathrm{AgO})^{-} /\left(\mathrm{TiO}_{2}\right)^{0} p$-type interface in the $\mathrm{AgTaO}_{3} / \mathrm{SrTiO}_{3}(001)$ heterostructure is presented. The $\mathrm{Ag} 4 d$ states strongly hybridize with the $\mathrm{O} 2 p$ states and contribute to the hole gas. It is demonstrated that the holes are confined to an ultra thin layer $(\sim 4.9 \AA)$ with a considerable carrier density of $\sim 10^{14} \mathrm{~cm}^{-2}$. We estimate a hole mobility of $18.6 \mathrm{~cm}^{2} \mathrm{~V}^{-1} \mathrm{~s}^{-1}$, which is high enough to enable device applications. (C) 2012 American Institute of Physics. [http://dx.doi.org/10.1063/1.4719106]
\end{abstract}

Epitaxial interfaces (IFs) of perovskite oxides are of eminent interest for possible device applications due to their fascinating and tunable properties. ${ }^{1-5}$ Very high mobility $\left(10^{4} \mathrm{~cm}^{2} \mathrm{~V}^{-1} \mathrm{~s}^{-1}\right)$ and considerable charge carrier density $\left(3.3 \times 10^{14} \mathrm{~cm}^{-2}\right)$ have been observed for the $\left(\mathrm{TiO}_{2}\right)^{0} /(\mathrm{LaO})^{+}$ $n$-type (electron doped) IF between the two band insulators $\mathrm{LaAlO}_{3}$ and $\mathrm{SrTiO}_{3}(\mathrm{STO}),{ }^{6}$ while the $\left(\mathrm{AlO}_{2}\right)^{-} /(\mathrm{SrO})^{0}$ (hole doped) $p$-type IF is insulating. The electronic properties of the $p$-type IF as a function of the oxygen vacancy concentration have been obtained theoretically by Park et al., ${ }^{7}$ consistent with the experimental situation. ${ }^{8}$ Experiments have revealed a band gap for the $(\mathrm{NaO})^{-} /\left(\mathrm{TiO}_{2}\right)^{0} p$-type IF in $\mathrm{NaNbO}_{3} / \mathrm{STO},{ }^{9}$ whereas it has been found theoretically that metallicity can be induced in this system, depending on the IF configuration and the layer ratio. ${ }^{10}$ The $p$-type IFs in $\mathrm{KTaO}_{3} / \mathrm{STO}, \mathrm{NaTaO}_{3} /$ $\mathrm{STO}$, and $\mathrm{LaGaO}_{3} / \mathrm{STO}$ show a metallic behavior. ${ }^{11-13}$

In polar heterostructures (HSs), a general issue is the thickness of the two-dimensional gas induced at the IF, which is usually some $1-2 \mathrm{~nm} .{ }^{14,15}$ For example, it has been argued that an ultra small thickness of the two-dimensional hole gas is important for enhancing the spin Hall and Rashba effects. ${ }^{16,17}$ Highly electronegative A site cations can help to tune this thickness. For this reason, we perform a systematic theoretical investigation of the IFs between the perovskite oxides $\mathrm{AgTaO}_{3}$ and STO and compare the results to related HSs.

The full-potential linearized augmented plane-wave method of density functional theory (WIEN2k package ${ }^{18}$ ) is employed, where the generalized gradient approximation is used for the exchange-correlation functional. Relativistic effects are taken into account fully for the core states, whereas the scalar relativistic approximation is used for the valence states, i.e., the spin-orbit coupling is neglected. For the wave function expansion inside the atomic spheres a maximum value of $\ell_{\max }=12$ and a plane-wave cutoff of $R_{m t} K_{\max }=7$ with $G_{\max }=24$ are applied. In the self-consistency calculations, a $11 \times 11 \times 2 \mathrm{k}$-space grid comprising 42 points within the irreducible wedge of the Brillouin zone is employed, and a total energy convergence of $10^{-4} \mathrm{Ry}$ is enforced to ensure well converged results. The structure is fully optimized by relaxing the atomic forces.

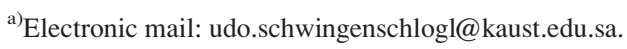

The bulk electronic structures of the compounds $\mathrm{AgTaO}_{3}$ and STO are well understood. ${ }^{19,20}$ They crystallize in cubic structures (space group No. 221, $P m \overline{3} m$ ) with experimental lattice constants of 3.948 and $3.905 \AA$, respectively. ${ }^{6,22}$ Hence, the lattice mismatch is only $1.1 \%$. The experimental band gaps of $\mathrm{AgTaO}_{3}$ and STO amount to 3.2 and $3.4 \mathrm{eV}$, respectively. ${ }^{6,23}$ Due to the generalized gradient approximation, the calculated band gaps are only 1.79 and $1.89 \mathrm{eV}$, in agreement with earlier band structure calculations. ${ }^{19,20}$ The $\mathrm{AgTaO}_{3} / \mathrm{STO}$ HS is modeled by means of the supercell approach, ${ }^{21}$ using an average value of the $\mathrm{AgTaO}_{3}$ and STO lattice constants for lattice matching. The supercell consists of 6 unit cells of $\mathrm{AgTaO}_{3}(\sim 2.4 \mathrm{~nm}$ thick) and 10 unit cells of STO $(\sim 4 \mathrm{~nm}$ thick), stacked along the $(001)$ direction. Three-dimensional periodic boundary conditions apply. Both the $\mathrm{TiO}_{2}$ terminated $(\mathrm{AgO})^{-} /\left(\mathrm{TiO}_{2}\right)^{0} p$-type and $\mathrm{SrO}$ terminated $\left(\mathrm{TaO}_{2}\right)^{+} /(\mathrm{SrO})^{0} n$-type IFs are realized in this supercell (compare Fig. 1). For $\mathrm{TiO}_{2}(\mathrm{SrO})$ termination holes (electrons) are transferred at the IF, which is known from other HSs. For polar systems, the electronic behavior depends critically on structural details in the IF region ${ }^{24,25}$ making a structure optimization essential. However, the obtained small modifications of the chemical bonding do not alter the physical picture in our case. The $\mathrm{Ti}-\mathrm{O}$ and $\mathrm{Ta}-\mathrm{O}$ bond lengths ( $1.96 \AA$ in the bulk) shrink slightly to $1.95 \AA$, whereas the $\mathrm{Sr}-\mathrm{O}$ and $\mathrm{Ag}-\mathrm{O}$ bond lengths $(2.78 \AA$ in the bulk) stay virtually the same. The good lattice match and small structural relaxation imply a high probability of epitaxial growth for the $\mathrm{AgTaO}_{3} / \mathrm{STO} \mathrm{HS}$.

Figure 2 depicts calculated partial densities of states (DOSs) of the $(\mathrm{AgO})^{-} /\left(\mathrm{TiO}_{2}\right)^{0} p$-type IF for projections on the $\mathrm{Ti}, \mathrm{Ta}, \mathrm{Ag}$, and $\mathrm{O}$ atoms next to the IF. A metallic state is evident, mainly due to the $\mathrm{O} 2 p$ states in the $\mathrm{TiO}_{2}$ layer but with considerable contributions of the $\mathrm{O} 2 p$ states in the

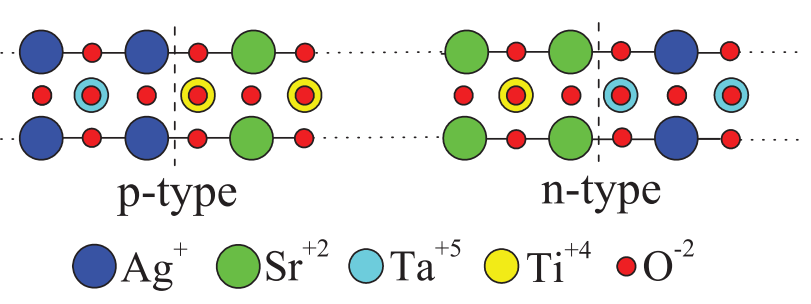

FIG. 1. $\mathrm{AgTaO}_{3} / \mathrm{STO}$ supercell with $\mathrm{TiO}_{2}$ terminated $p$-type and $\mathrm{SrO}$ terminated $n$-type IFs. 

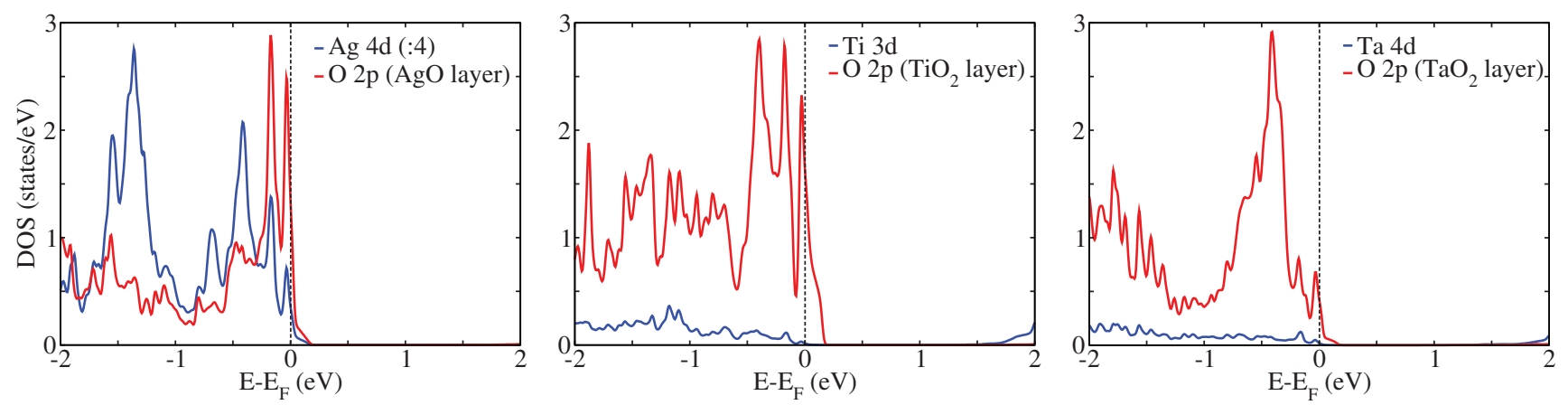

FIG. 2. Projected DOSs at the fully relaxed $\mathrm{AgTaO}_{3} / \mathrm{STO} p$-type IF. The $\mathrm{Ag} 4 d$ DOS is down-scaled by a factor 4 for clarity.

adjacent $\mathrm{AgO}$ and $\mathrm{TaO}_{2}$ layers. Charge redistribution at the IF is established by the fact that the $\mathrm{O} 2 p$ states shift significantly to higher energy and become partially unoccupied, which leaves the IF metallic. Interestingly, the Ag $4 d$ states also shift to higher energy and contribute remarkably to the metallic IF. Such a crossing of the Fermi energy $\left(E_{F}\right)$ is not observed for other polar IFs with less electronegative A site cations. Note the strong hybridization between the $\mathrm{O} 2 p$ and $\mathrm{Ag} 4 d$ states near $E_{F}$. The IF Ti $3 d$ and Ta $5 d$ states (located right next to the $\mathrm{AgO}$ layer) do not contribute to the metallicity (see Fig. 2). The same holds true for the Ag $4 d$ states in the third layer from the IF. Most strikingly, the charge transfer occurs only in a very narrow region confined by the first $\mathrm{AgO}$ and $\mathrm{TiO}_{2}$ layers. Therefore, the spatial extension of the two-dimensional hole gas amounts to only $4.9 \AA$, which is much smaller than in related HSs. We have checked that there is no interaction between the two IFs in our supercell which could invalidate these results.

The Ti $3 d$, Ta $5 d$, and $\mathrm{O} 2 p$ partial DOSs for the $\left(\mathrm{TaO}_{2}\right)^{+} /(\mathrm{SrO})^{0} n$-type IF are given in Fig. 3. We find metallicity, which mainly comes from the Ti $3 d$ and Ta $5 d$ states with very small $\mathrm{O} 2 p$ contributions from atoms in the $\mathrm{TaO}_{2}$ and $\mathrm{SrO}$ IF layers. Due to charge transfer the Ti $3 d$ and Ta $5 d$ states shift to lower energy and become partially occupied, where the shift of the Ta $5 d$ states is considerably larger than that of the Ti $3 d$ states. Therefore, the Ta $5 d$ states are primarily responsible for the IF metallicity, but considerable contributions of the Ti atoms next to the SrO IF layer cannot be neglected. The DOS also demonstrates a significant hybridization between the Ti $3 d$ and Ta $5 d$ states around $E_{F}$.

By the octahedral crystal field, the Ti $3 d$ and Ta $5 d$ states split into $d_{3 z^{2}-r^{2}}, d_{x y}, d_{x^{2}-y^{2}}$ and degenerate $d_{x z}+d_{y z}$ symmetry components. The metallicity originates from the $d_{x y}$ and $d_{x z}+d_{y z}$ states, where the $d_{x y}$ orbitals are lower in energy similar to $\mathrm{LaAlO}_{3} / \mathrm{STO} .{ }^{26,27}$ The $d_{3 z^{2}-r^{2}}$ and $d_{x^{2}-y^{2}}$ states remain unoccupied as they overlap directly with $\mathrm{O} 2 p$ orbitals and, hence, appear at higher energy. Ag does not contribute to the metallicity in this case. In general, the charge transfer is strongest within the first $\mathrm{TaO}_{2}$ layer and within the $\mathrm{TiO}_{2}$ layer next to the $\mathrm{SrO}$ IF layer. It declines rapidly away from the IF. The second $\mathrm{TaO}_{2}$ layer is on the edge of metallicity, while all atoms further away show an insulating state. On the $\mathrm{SrO}$ side, metallic states are found up to the third $\mathrm{TiO}_{2}$ layer. Therefore, the total width of the metallic region perpendicular to the IF amounts to $15.8 \AA$, which is a typical value.

Evaluation of the orbital occupations yields for the $p$-type IF O $2 p$ hole counts of $0.05,0.18$, and 0.02 in the $\mathrm{AgO}, \mathrm{TiO}_{2}$, and $\mathrm{TaO}_{2}$ layers, respectively, and thus charge carrier densities of $3.2 \times 10^{13} \mathrm{~cm}^{-2}, 11.7 \times 10^{13} \mathrm{~cm}^{-2}$, and $1.6 \times 10^{13} \mathrm{~cm}^{-2}$. Moreover, the Ag $4 d$ orbitals contain 0.06 holes, which corresponds to a significant carrier density of $4.9 \times 10^{13} \mathrm{~cm}^{-2}$. Similarly, for the $n$-type IF we find in the Ti $3 d$ and Ta $5 d$ orbitals in the vicinity of the IF 0.07 and 0.10 electrons, respectively. This corresponds to carrier densities of $4.5 \times 10^{13} \mathrm{~cm}^{-2}$ and $6.6 \times 10^{13} \mathrm{~cm}^{-2}$. The carrier density in $\mathrm{AgTaO}_{3} / \mathrm{STO}$, therefore, is much higher than in $\mathrm{KTaO}_{3} / \mathrm{STO}$ (Ref. 12) as well as in the $n$-type IF of $\mathrm{LaAlO}_{3} /$ STO at low temperature. ${ }^{14,27,28}$

To achieve a deeper understanding of the special properties of the $(\mathrm{AgO})^{-} /\left(\mathrm{TiO}_{2}\right)^{0} p$-type IF, calculated band structures are shown in Fig. 4. A substantial hybridization between the $\mathrm{Ag} 4 d$ and $\mathrm{O} 2 p$ states is observed, in particular at the $\mathrm{X}$ point between the $\mathrm{Ag} d_{3 z^{2}-r^{2}}$ and $\mathrm{O} p_{x}, p_{y}$ states (from atoms in the $\mathrm{AgO}$ layer) and at the $\Gamma$ point between the $\mathrm{Ag} d_{x y}+d_{y z}$ and $\mathrm{O} p_{x}, p_{y}$ states (from atoms in the $\mathrm{TiO}_{2}$
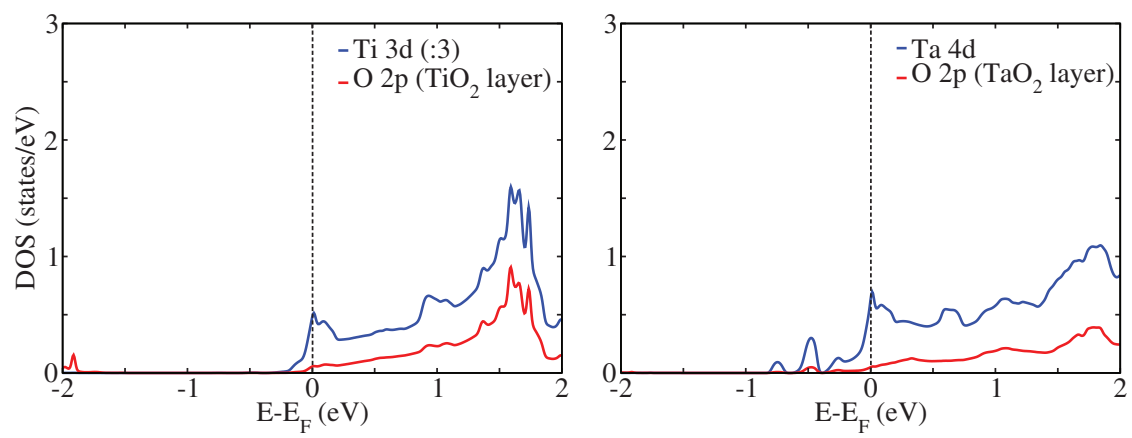

FIG. 3. Projected DOSs at the fully relaxed $\mathrm{AgTaO}_{3} / \mathrm{STO} n$-type IF. The Ti $3 d$ DOS is downscaled by a factor 3 for clarity. 
layer), see Fig. 4. Both are not the case for the closely related $(\mathrm{KO})^{-} /\left(\mathrm{TiO}_{2}\right)^{0}$ and $(\mathrm{NaO})^{-} /\left(\mathrm{TiO}_{2}\right)^{0} p$-type IFs, where the A site cations $\mathrm{K}$ and $\mathrm{Na}$ do not contribute to the metallic IF states. ${ }^{10,11}$ In addition, at the $\mathrm{M}$ point the $\mathrm{O} p_{x}, p_{y}$ orbitals dominate. As discussed before, the $\mathrm{O} 2 p$ hole density in the $\mathrm{TiO}_{2}$ IF layer is much higher than the $\mathrm{Ag} 4 d$ and $\mathrm{O} 2 p$ hole densities in the $\mathrm{AgO}$ IF layer.

Effective band masses can be evaluated from the curvature of the bands in Fig. 4 via the relation $m^{*}=-\hbar^{2}$ $\left(d^{2} \epsilon / d k^{2}\right)^{-1}$ and then used for estimating the hole mobility. Applying the one-band approximation, since there are no degenerate bands, the effective mass is obtained from the curvatures at the extreme points. The band extrema are fitted by parabolic curves for this purpose. The contributions from the high symmetry points $\mathrm{X}$ and $\mathrm{M}$ lead to the hole effective mass $m^{*}=-3.32 m_{e}$, where $m_{e}$ is the mass of the bare electron. The negative sign here reflects that we deal with holes. Our calculated $m^{*}$ is similar in magnitude to data for $\mathrm{LaAlO}_{3} / \mathrm{STO},{ }^{14,15}$ which has important implications. For example, the thermoelectric power is high because the gain in the Seebeck coefficient is quadratic in $m^{*}$ while the loss in the mobility is linear. ${ }^{29}$ Hole mobilities at zero Kelvin can be calculated from the relation $\mu=e \tau / m^{*}$, where the relaxation time $\tau$ for $\mathrm{AgTaO}_{3} / \mathrm{STO}$ is assumed to be $35 \mathrm{fs}$, following estimations in the literature. ${ }^{30,31} \mathrm{We}$ obtain for our hole gas $\mu=18.6 \mathrm{~cm}^{-2} \mathrm{~V}^{-1} \mathrm{~s}^{-1}$, which is a similar magnitude as reported for the highly mobile electron gas in $\mathrm{LaTiO}_{3} /$ STO. ${ }^{30}$

The confinement of the metallic states to a very narrow region around the $p$-type IF, as compared to related HSs such as $\mathrm{KTaO}_{3} / \mathrm{STO}, \mathrm{NaTaO}_{3} / \mathrm{STO}$, and $\mathrm{NaNbO}_{3} / \mathrm{STO}$, is due to the high electronegativity of Ag. In order to further highlight the ultra small thickness, an isosurface plot of the hole density is shown in Fig. 5 and compared to analogous results for the $(\mathrm{KO})^{-} /\left(\mathrm{TiO}_{2}\right)^{0} p$-type IF on the right hand side of the figure. The spatial extension of the metallic region is clearly reduced in the presence of $\mathrm{Ag}$, since its high electronegativity results in an enhanced covalent bonding with the surrounding $\mathrm{O}$ atoms and, consequently, a suppression of the long range charge transfer. Figure 5 demonstrates that the $\mathrm{Ag} \mathrm{IF}$ atoms contribute to the hole gas due to hybridization

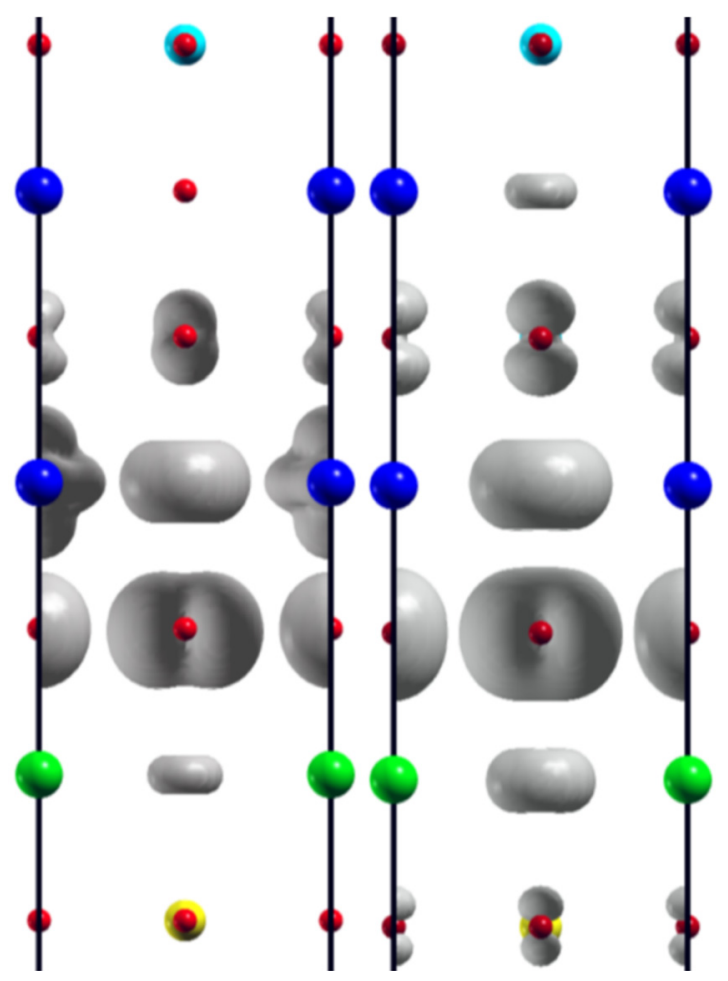

FIG. 5. Isosurface plots for the conduction band $\left(E_{F}\right.$ to $\left.0.5 \mathrm{eV}\right)$ at the $\mathrm{AgTaO}_{3} / \mathrm{STO}$ (left) and $\mathrm{KTaO}_{3} / \mathrm{STO}$ (right) $p$-type IFs. The same color code as in Fig. 1 is used.

between the $\mathrm{Ag} 4 d$ and $\mathrm{O} 2 p$ states, whereas the $\mathrm{K}$ atoms do not contribute. Moreover, as a consequence of the covalent bonding between $\mathrm{Ag}$ and $\mathrm{O}$, a high hole mobility is expected. This explains the findings described above. We have confirmed our results by calculations employing the self interaction correction with an onsite interaction of $4 \mathrm{eV}$ (Ref. 32) on the $\mathrm{Ti}$ and $\mathrm{Ta}$ atoms. In addition, we have checked whether intermixing of $\mathrm{Ti}$ and $\mathrm{Ta}$ or $\mathrm{Sr}$ and $\mathrm{Ag}$ alters our conclusions. However, in both cases the energy increases (by $0.81 \mathrm{eV}$ and $0.59 \mathrm{eV}$, respectively) and intermixing can be excluded, in contrast to other IFs. ${ }^{33}$

In conclusion, the electronic properties of the $\mathrm{AgTaO}_{3} /$ STO HS have been investigated by density functional theory.
$\operatorname{Ag} 4 d$

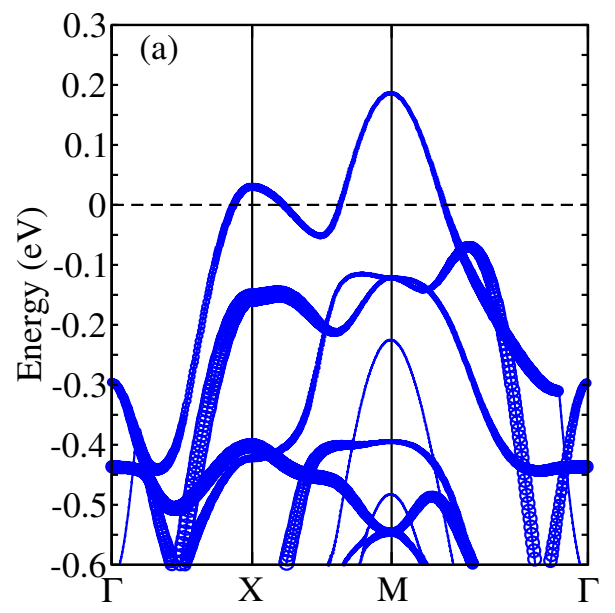

O $2 p$ (AgO layer)

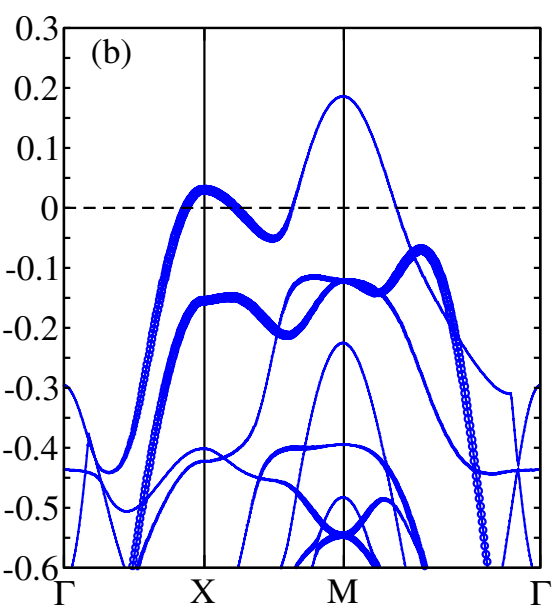

$\mathrm{O} 2 \mathrm{p}\left(\mathrm{TiO}_{2}\right.$ layer $)$

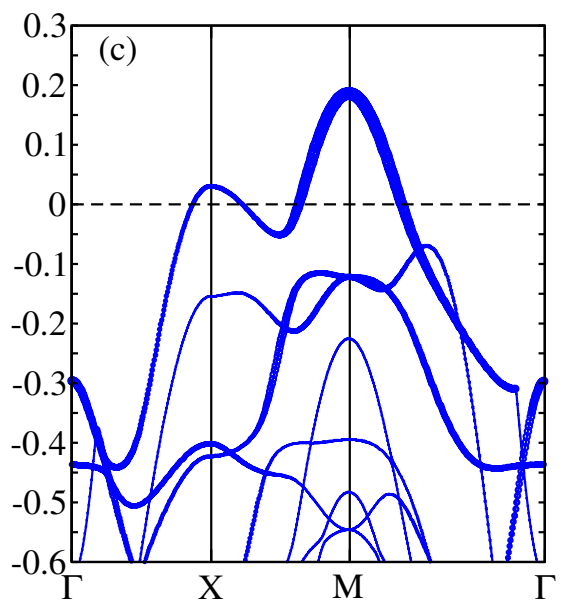

FIG. 4. Orbitally weighted band structures for the $p$-type $\mathrm{AgTaO}_{3} / \mathrm{STO}$ IF. 
Metallic states are found for both the $(\mathrm{AgO})^{-} /\left(\mathrm{TiO}_{2}\right)^{0} p$-type IF and $\left(\mathrm{TaO}_{2}\right)^{+} /(\mathrm{SrO})^{0} n$-type IF. At the $n$-type IF, the metallic region spans over a wide range ( $15.8 \AA$ thickness). On the contrary, covalent bonding between the $\mathrm{Ag} 4 d$ and $\mathrm{O} 2 p$ states at the $p$-type IF leads to a confinement of the hole gas to a very narrow region $(\sim 4.9 \AA$ thickness $)$ around the $\mathrm{IF}$. The hole gas is not only very thin but also shows a high carrier density. Furthermore, the calculated hole mobility of $18.6 \mathrm{~cm}^{2} \mathrm{~V}^{-1} \mathrm{~s}^{-1}$ is comparable in value with the mobility of the electron gas in $\mathrm{LaTiO}_{3} / \mathrm{STO}$. This high value is attributed to the participation of the $\mathrm{Ag} 4 d$ states in the metallicity. It makes $\mathrm{AgTaO}_{3} / \mathrm{STO}$ and related HSs, such as $\mathrm{AgNbO}_{3} / \mathrm{STO},{ }^{34}$ strong candidates for nanoscale oxide device applications.

Fruitful discussions with H. N. Alshareef and support by KAUST supercomputing are gratefully acknowledged.

${ }^{1}$ S. A. Pauli and P. R. Willmott, J. Phys.: Condens. Matter 20, 264012 (2008). ${ }^{2}$ R. Pentcheva and W. E. Pickett, J. Phys.: Condens. Matter 22, 043001 (2010).

${ }^{3}$ Q.-H. Wu, Crit. Rev. Solid State Mater. Sci. 36, 1 (2011).

${ }^{4}$ J. M. Rondinelli and N. A. Spaldin, Adv. Mater. 23, 3363 (2011).

${ }^{5}$ P. Zubko, S. Gariglio, M. Gabay, P. Ghosez, and J.-M. Triscone, Annu. Rev. Condens. Matter Phys. 2, 141 (2011).

${ }^{6}$ A. Ohtomo and H. Y. Hwang, Nature (London) 427, 423 (2004).

${ }^{7}$ M. S. Park, S. H. Rhim, and A. J. Freeman, Phys. Rev. B 74, 205416 (2006).

${ }^{8}$ N. Nakagawa, H. Y. Hwang, and D. A. Muller, Nat. Mater. 5, 204 (2006).

${ }^{9}$ J. Narkilahti, M. Plekh, J. Levoska, and M. Tyunina, Phys. Rev. B 79, 014106 (2009).

${ }^{10}$ R. Oja and R. M. Nieminen, Phys. Rev. B 80, 205420 (2009).

${ }^{11}$ S. Nazir, N. Singh, and U. Schwingenschlögl, Phys. Rev. B 83, 113107 (2011).

${ }^{12}$ S. Nazir and U. Schwingenschlögl, Appl. Phys. Lett. 99, 073102 (2011).

${ }^{13}$ S. Nazir, N. Singh, and U. Schwingenschlögl, Appl. Phys. Lett. 98, 262104 (2011).

${ }^{14}$ A. Dubroka, M. Rössle, K. W. Kim, V. K. Malik, L. Schultz, S. Thiel, C. W. Schneider, J. Mannhart, G. Herranz, O. Copie, M. Bibes, A. Barthélémy, and C. Bernhard, Phys. Rev. Lett. 104, 156807 (2010).
${ }^{15}$ P. Delugas, A. Filippetti, V. Fiorentini, D. I. Bilc, D. Fontaine, and P. Ghosez, Phys. Rev. Lett. 106, 166807 (2011).

${ }^{16}$ J. Wunderlich, B. Kaestner, J. Sinova, and T. Jungwirth, Phys. Rev. Lett. 94, 047204 (2005).

${ }^{17}$ C. H. Yang, W. Xu, Z. Zeng, F. Lu, and C. Zhang, Phys. Rev. B 74, 075321 (2006).

${ }^{18}$ P. Blaha, K. Schwarz, G. Madsen, D. Kvasicka, and J. Luitz, WIEN2k, Technical University of Vienna, Vienna, 2001.

${ }^{19}$ S. Cabuk and S. Simsek, Cent. Eur. J. Phys. 6, 730 (2008).

${ }^{20}$ M. Djermouni, A. Zaoui, S. Kacimi, and B. Bouhafs, Comput. Mater. Sci. 49, 904 (2010).

${ }^{21}$ M. U. Kahaly, S. Nazir, and U. Schwingenschlögl, Appl. Phys. Lett. 99, 123501 (2011).

${ }^{22}$ G. E. Kugel, M. D. Fontana, M. Hafid, K. Roleder, A. Kania and, M. Pawelczyk. J. Phys. C 20, 1217 (1987).

${ }^{23}$ H. Kato, H. Kobayashi, and A. Kudo, J. Phys. Chem. B 106, 12441 (2002).

${ }^{24}$ U. Schwingenschlögl and C. Schuster, Chem. Phys. Lett. 467, 354 (2009); Europhys. Lett. 81, 17007 (2008).

${ }^{25}$ U. Schwingenschlögl and C. Schuster, Europhys. Lett. 86, 27005 (2009).

${ }^{26}$ M. Salluzzo, J. C. Cezar, N. B. Brookes, V. Bisogni, G. M. De Luca, C. Richter, S. Thiel, J. Mannhart, M. Huijben, A. Brinkman, G. Rijnders, and G. Ghiringhelli, Phys. Rev. Lett. 102, 166804 (2009).

${ }^{27}$ M. Huijben, G. Rijnders, D. H. A. Blank, S. Bals, S. V. Aert, J. Verbeeck, G. V. Tendeloo, A. Brinkman, and H. Hilgenkamp, Nat. Mater. 5, 556 (2006).

${ }^{28}$ Ariando, X. Wang, G. Baskaran, Z. Q. Liu, J. Huijben, J. B. Yi, A. Annadi, A. R. Barman, A. Rusydi, S. Dhar, Y. P. Feng, J. Ding, H. Hilgenkamp, and T. Venkatesan, Nat. Commun. 2, 188 (2011).

${ }^{29}$ W. Wunderlich, H. Ohta, and K. Koumoto, Phys. B: Condens. Matter 404, 2202 (2009).

${ }^{30}$ S. S. A. Seo, W. S. Choi, H. N. Lee, L. Yu, K. W. Kim, C. Bernhard, and T. W. Noh, Phys. Rev. Lett. 99, 266801 (2007).

${ }^{31}$ K. Koumoto, Y. Wang, R. Zhang, A. Kosuga, and R. Funahashi, Annu. Rev. Mater. Res. 40, 363 (2010).

${ }^{32}$ R. Arras, V. G. Ruiz, W. E. Pickett, and R. Pentcheva, Phys. Rev. B 85, 125404 (2012).

${ }^{33}$ S. A. Chambers, M. H. Engelhard, V. Shutthanandan, Z. Zhu, T. C. Droubay, L. Qiao, P. V. Sushko, T. Feng, H. D. Lee, T. Gustafsson, E. Garfunkel, A. B. Shah, J.-M. Zuo, and Q. M. Ramasse, Surf. Sci. Rep. 65, 317 (2010).

${ }^{34}$ P. Sciau, A. Kania, B. Dkhil, E. Suard, and A. Ratuszna, J. Phys.: Condens. Matter 16, 2795 (2004). 